\title{
Reflections of the
} past

\section{Ian Stewart}

Felix Klein and Sophus Lie: Evolution of the Idea of Symmetry in the Nineteenth Century. By I. M. Yaglom. Translated by Sergei Sossinsky. Birkhäuser: 1987. Pp.237. \$40.

KLEIN and Lie, two giants of the nineteenth century, were between them responsible for deep-seated changes in the way mathematicians think about geometry. For Euclid there was only one geometry, the geometry of physical space. By the time of Bolyai, Gauss and Lobachevskii, Euclid's had become just one of three distinct possible kinds of geometry. Soon three had become dozens; then, in the hands of Bernhard Reimann, an infinity. To control this proliferation, some general organizing principles were needed. They were supplied by Klein and developed into a deep and marvellous theory by Lie. Before them, geometry was the study of shapes in space; after them it was the study of invariants of transformation groups.

For example, consider the concept of an isosceles triangle. For Euclid, this is a figure made up from three straight lines, two of which have the same length. Its other properties, such as two angles being equal, are proved by logical arguments based on a small number of fundamental axioms. To Klein, on the other hand, it is not the triangle that is important, but the rigid motions of the plane that preserve its shape and size: rotations, reflections, translations. The equality of two sides is a consequence of the triangle's mirror symmetry about a suitable line; and so is the equality of the corresponding angles. Complicated exercises in logic become transparent applications of symmetry.

Thus the concepts of Euclid's geometry are reformulated as quantities, such as lengths and angles, that remain unchanged under some collection of transformations. These transformations are said to form a group, which means that the result of applying any two of them in turn is just another permitted transformation. Distinct geometries become distinct groups: the surface features are suppressed and the underlying abstract structure is brought to the fore.

Felix Klein and Sophus Lie is an attempt to describe the mathematics involved in this development, and the people behind it. This is an ambitious task, and to some extent the book falls between two stools. On the one hand, it is an authoritative account of an extremely important series of developments that changed the face of mathematics, and most of it is accessible

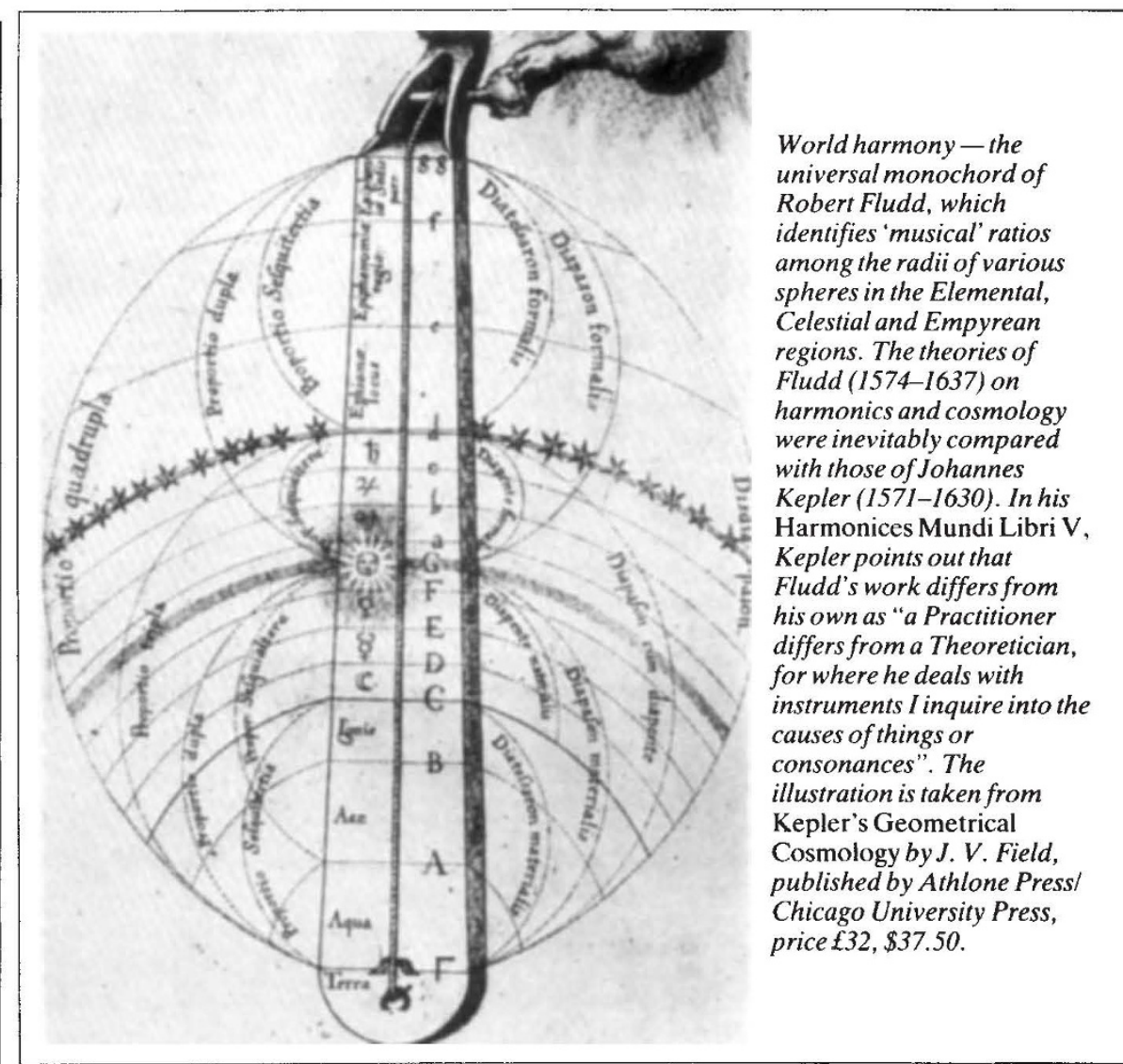

to a non-specialist. On the other hand, the choice of material is more than a little idiosyncratic, the contents do not adequately reflect the title or the subtitle, and there are passages that are far too technical for the non-specialist. It is also rather fragmented.

Taken at face value, the book deals with the discovery of group theory and noneuclidean geometries. It takes up the story around the time of Evariste Galois, who tackled the general question "When is a polynomial equation soluble?". There is a brief excursion into the geometric works of Darboux and Plücker, after which the story drops back a century or so to the early days of projective geometry. The tangled tale of Gauss, Lobachevskii and Bolyai leads, via non-euclidean geometry, into hypercomplex numbers and the foundations of vector algebra. There follows a chapter on Lie and another on Klein's Erlangen programme - effectively the statement that geometry is group theory. A final chapter adds some biographical details.

This occupies the first 137 pages. There follow another hundred pages of notes, dotting the i's, crossing the t's and filling in the technicalities. The history is sound, the mathematics important, the exposition accurate - but the overall effect is unsatisfactory. For example the chapter that is supposed to describe Lie's theory of continuous groups (now called Lie groups) is in fact devoted to the formal properties of associative algebras, leading up to the concept of a Lie algebra. Two pages of small print are spent describing the classical families of simple Lie groups in terms that require far more mathematical maturity than the surrounding text. The small print is intended as a warning, but footnotes are surely a more appropriate place for such material. A reader who does not already know will get little idea of what a Lie group is; one searches in vain for a clear indication even of what the word 'continuous' is supposed to mean in this context. And accessible examples that emphasize the role of continuity, such as the rotation group in three-dimensional space, are absent.

The reader might also be forgiven for emerging with the belief that the main reason why Lie groups are central to today's mathematics is that they generalize Galois Theory to differential equations, when in fact this is of relatively minor importance compared to their role in topology, dynamics and indeed group theory itself. There is also remarkably little said, anywhere, about symmetry the subtitle notwithstanding.

The notes may well be the book's saving grace; one can learn a lot from them. They include a fascinating discussion of mathematical anti-Semitism, a page on Cayley's 'octaves' (or octonions as they tend to be called now) and a brief history of vector calculus. Maybe that is the point - this is not really a book, but a set of notes for a book.

Ian Stewart is a Reader in the Mathematics Institute, University of Warwick, Coventry CV4 $7 A L, U K$. 